\title{
Gender Classification using Geometric Facial Features
}

\author{
Swathi Kalam \\ Department of Computer Science \& Technology \\ VR Siddhartha Engineering College
}

\author{
Geetha Guttikonda \\ Department of Information Technology \\ VR Siddhartha Engineering College
}

\begin{abstract}
Gender classification has become an essential task in human computer interaction (HCI). Gender classification is used in immense number of applications like passive surveillance, control in smart buildings (restricting access to certain areas based on gender) and supermarkets, gender advertising, security investigation. So far detection of gender using facial features is done by using the methods like Gabor wavelets, artificial neural networks and support vector machine. In this work, facial distance measure is used as a progenitor to achieve the gender classification. The proposed approach performs gender classification using mathematical operations on the frontal pose face images using Matlab. This work can be further evaluated in future by using different databases with various poses other than the frontal pose.
\end{abstract}

\section{Keywords}

Gender classification, feature extraction

\section{INTRODUCTION}

Gender means the range of physical, biological, mental and behavioral characteristics which differentiates masculinity and femininity. Classification means categorization. Gender classification is a binary classification technique to classify male and female. This technique has become a most important task as it has many applications. So far the work is used to recognize the gender by visual observation but now the work is emphasized to a computer so that it can guess whether the given is a male or female.

The general definition for gender classification is the genetic or environmental process which is used to specify an individual characteristic physically [1]. Gender detection is one of the most interesting and challenging field in the biometrics. The recognition process can be more efficient if it is based on features that provide some information about the class to be detected. Generally it is defined as the genetic process by which the gender of an individual is determined based on their facial features.

From last 30 years experimental research work has been conducted in this field as a result the current position of facial gender detection is on well progress. Automatic gender recognition problem is essential in places like supermarkets, restaurants and also where security through surveillance is desired. Hence, it is necessary in computer aided systems where human interaction is required. The gender of an individual is categorized by visual observation [12] of images but it is difficult for a computer for differentiating the gender. So the gender recognition is done by taking into consideration features viz., distances of eye, nose, lips and chin.

\section{RELATED WORK}

The first automatic system for detecting face and gender was developed by Moghaddam and Yang [2] had used maximumlikelihood estimation for detection of face and facial features. For classifying gender several classification algorithms and the experiments were done by considering the set of FERET images. The most fascinating results in this paper were that the performance of Support Vector Machine (SVM) is better when compared to the other classifiers and the classification level of SVM did not affect the resolution of the face.

Shakhnarovich et al. [3] developed a system that combines the cascaded face detector system which was developed by Viola and Jones with discrete Adaboost-based gender and ethnicity classification. The main advantage of this system is that many of the preprocessing and calculations which have to be done can be shared by both the face detector and the gender classifier. Visual gender classification for low resolution images was proposed by Ming-Hsuan Yang and Baback Moghaddam [4] They considered thumbnail faces (21x12 pixels) processed from 1755 images from the FERET face database by using SVM classifier and related the performance accuracy of human test issues on high and low resolution images.

Baback Moghaddam and Ming-Hsua [5] proposed an appearance based method for identifying gender from facial images using nonlinear SVM classifier and compared the results with traditional classifiers and modern techniques such as Radial Basis Function (RBF) networks and large ensemble-RBF classifiers, While comparing the difference the performance with low-resolution and their corresponding higher resolution images is very less during classification.

Zehang Sun et al., [6] proposed Gender classification from frontal face images using genetic feature subset selection. Here they used Principal Component Analysis (PCA) to represent each individual image as a feature vector in a low dimensional space and based on Genetic algorithms, a subset of features are extracted from the low-dimensional representation by ignoring certain eigenvectors which are not needed for classifying facial feature information. Classifiers like Bayes, Neural Network, SVM, and LDA are used and compared by using Genetic Algorithm feature subset selection. Roytatsu Iga et al., [7] proposed an algorithm for categorizing gender and age using SVM classifier by considering the features like geometric alignment and brightness of facial images. For the position detection of the face graphical matching method along with GWT is used. GWT features like geometric arrangement of color, hair and mustache are taken into consideration for the classification of gender and for age classification texture spots, wrinkles and flabs of skin are considered.

Multi-view gender classification by focusing on shape and texture data for representing facial images was proposed by HuiChing Lain and Buo-Liang Lu [8]. In this, the facial area is divided into small regions by using local binary pattern (LBP) histograms. Gender classification using Shape from Shading (SFS)was proposed by Jing Wu et al., [9].Based on the Principal Geodesic Analysis parameters Linear Discriminant Analysis (LDA) discriminate female and male of the test faces. SFS technique is used to improve the

performance analysis of gray scale face images regarding classification

ErnoMakinen and Roope Raisamo [10] had done the work on experimental assessment of gender classification. This study performs comparison on the four major gender classification and 
automatic alignment methods with non-aligned faces and faces which are manually aligned. They also studied how the classification precision was aff ected when resizing of face image is done before or after alignment. Finally, they performed a keen analysis for the classifiers by performing geometric transformations like rotation, scale, and translation of the face images.

A Neural Network based frontal face detection system that performs classification using facial information was proposed by Mallikarjuna Rao et al., [11]. The consistency of this method depends on Pixel and geometric facial features. The toughness of classification is depended on pi-sigma neural network and the cyclic shift invariance techniques.

\section{MODEL}

The gender classification problem can be solved by a 3-step process. The first is to preprocess an image second step is to extract features and third is to classify gender. Similar to the face detection task, the gender classification task also considered as a binary classification but now with the result being male or female instead of face or non-face.

Essentially, gender classification consists of 3 main steps: (1) preprocessing (2) feature extraction, (3) classification

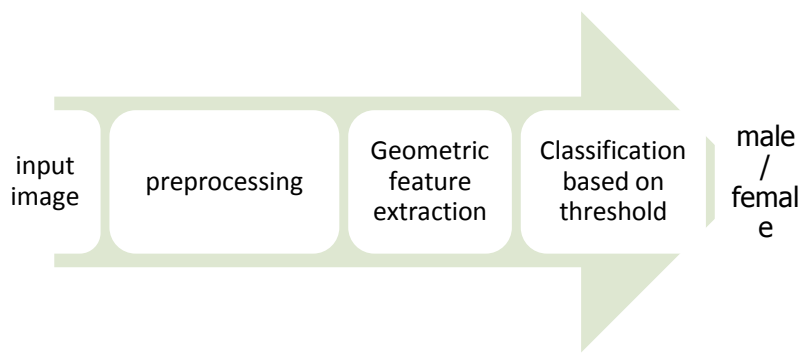

Figure 1: Steps for Gender Classification

\subsection{Preprocessing}

Pre-processing of a face image will undergo several processes in order to attain a transformed face image which in turn enhances the quality of the face image by holding noticeable features of the visual quality. Color conversion Noise reduction, and edge detection are some pre-processing techniques. Before preprocessing the image is selected from pre-stored face database.

\subsubsection{Color Conversion}

An RGB color image is an MxNx3 collection of color pixels corresponding to the red, green and blue components which specify a specific spatial location. The range of values is determined by data class components. For example class double of an RGB image is in the range of values $[0,1]$ if it is class unit8 the range of values is $[0,255]$ and if it is unit 16 then the range will be [0, 65535].The bit depth of an RGB image is defined by number of bits corresponding to the pixel value of a part of an image. The possible colors in an RGB image are designated by $(2 b)^{3}$, where $b$ is the number of bits in each part of an image. For 2-bit case, the numbers of colors are64, for 8bit case the number of colors are $16,777,216$. In this three dimensional RGB image is converted into two dimensional gray scale images for easy processing of face image [12].

After performing color conversion resize the image into $256 \times 256$ for our convenience.

\subsubsection{Noise Reduction}

The process of removal of noise is called noise reduction. Noise is due to either some disturbance in mechanism of electronic devices or due some problem in algorithm. Images taken by using digital cameras will pick up noise from different sources. Noise may also be caused by typical sources like particles of dust inside the camera and overheated or from faulty CCD elements. For practical purpose noise will be partially removed.

The noise may be of different types like salt and pepper noise, Gaussian noise etc. Salt and pepper noise is caused due to sparse light and dark disturbances or varying difference in color with the surrounding pixels. Small numbers of image pixels are affected by the salt and pepper noise. Gaussian noise is the noise in which each pixel in the image will be changed from its original value by small amount.

There are two types of noise models. Additive noise and multiplicative noise depending on the modality used for image acquisition. The noise due to electronic components in the acquisition hardware will be modeled with Gaussian noise which is independent of data, the data dependent noise such as quantum noise in X-ray imaging is modeled with Poisson distribution [13].

$$
\begin{aligned}
& g(x, y)=f(x, y) * h(x, y)+\eta(x, y) \\
& \mathrm{g}(\mathrm{x}, \mathrm{y})=[\mathrm{f}(\mathrm{x}, \mathrm{y}) \mathrm{h}(\mathrm{x}, \mathrm{y})]+\mathrm{N}(\mathrm{x}, \mathrm{y}) \\
& \text { Where } g(x, y) \text { is the noisy and blurred observation, }
\end{aligned}
$$

$H$ is the blurring kernel and $f(x, \mathrm{y})$ is the Signal that is to be recovered. In the case of denoising problem the blurring kernel will be dropped and the degradation model will be given as

$$
\begin{aligned}
& g(x, y)=f(x, y)+\eta(x, y) \\
& g(x, y)=f(x, y)+N(x, y)
\end{aligned}
$$

In the case of multiplicative noise the model is given as

$$
g(x, y)=f(x, y) \cdot \eta(x, y)
$$

For noise removal, some filters like linear, nonlinear, adaptive filtering etc are used. Here median filter is used for noise removal. A median filter is an example of a non-linear filter which is very good at preserving image detail. A median filter is a rank-selection (RS) filter, a particularly harsh member of the family of rank-conditioned rank-selection (RCRS) filters. To run a median filter consider each pixel in the image and sort the neighboring pixels into order based upon their intensities, then replace the original value of the pixel with the median value from the list. It is mostly preferred, in photographic applications. Median and RCRS filters are good at removing salt and pepper noise from an image, and also cause relatively little blurring of edges.

\subsubsection{Edge Detection}

Edge Detection is mainly used for detecting and extracting the boundary features in a digital image. The detection is done at the place where the image brightness change sharply and also at the place where there will be discontinuities. The main reason of detecting sharp edges of images is to capture and observe the place where there is a change at a particular point which can change the entire incident. In an ideal case, the result of edge detector may lead to the formation of set of connected curves that corresponds to variations in surface orientation. Therefore, implementing edge detection algorithm on any image may significantly reduce the quantity of data to be processed and also removes the undesirable data 
Many edge detection techniques came into existence. These detection methods differ in the types of smoothing filters that are applied for calculating gradient estimates in the $\mathrm{X}$-axis and $\mathrm{Y}$ axis. A survey was held by Ziou and Tabbone in 1998 to know actually how number of edge detection techniques came into existence.

The development of the edge detection algorithm is done based on the following factors:

- Good detection - the algorithm should point or mark as many as real images that are possible.

- Minimal response - a given edge should mark only once and also only in possible way of an image; the noise of the image should not create false edge.

- Good localization - the marked edges must always be nearer to the real in any image.

\subsubsection{Edge Detection Techniques}

There are many edge detection techniques like Sobel, Prewitt, Robert cross, canny edge detector so on. Out of them canny edge detector for detecting edges is used.

Canny edge detector was developed by John F.Canny in1986. It is used as multi-stage algorithm to detect a wide range of edges in images. Initially the image is smoothed by Gaussian filter.

The algorithm runs in 5 separate steps:

1. Smoothing: Blurring of the image to remove noise.

$$
B=\frac{1}{115}\left[\begin{array}{ccccc}
2 & 4 & 5 & 4 & 2 \\
4 & 9 & 12 & 9 & 4 \\
5 & 12 & 15 & 12 & 5 \\
4 & 9 & 12 & 9 & 4 \\
2 & 4 & 5 & 4 & 2
\end{array}\right]
$$

2. Finding gradients: The edges should be marked where the gradients of the image has large magnitudes.

$$
\begin{gathered}
K G x=\left(\begin{array}{ccc}
-1 & 0 & 1 \\
-2 & 0 & 2 \\
-1 & 0 & 1
\end{array}\right) \\
K G y=\left(\begin{array}{ccc}
1 & 2 & 1 \\
0 & 0 & 0 \\
-1 & -2 & -1
\end{array}\right)
\end{gathered}
$$

3. Non-maximum suppression: Only local maxima should be marked as edges.

4. Double thresholding: Potential edges are determined by thresholding.

5. Edge tracking by hysteresis: Final edges are determined by suppressing all edges that are not connected to a very certain (strong) edge.

$$
|G|=\sqrt{G x^{2}+G y^{2}}
$$

$$
\begin{gathered}
|G|=|G x|+|G y| \\
\theta=\arctan \left(\frac{|G x|}{|G y|}\right)
\end{gathered}
$$

Gradient along $\mathrm{x}$ direction and y direction is calculated as

$$
\begin{aligned}
& G x=f(x+1, y)-f(x, y) \\
& G y=f(x, y+1)-f(x, y)
\end{aligned}
$$

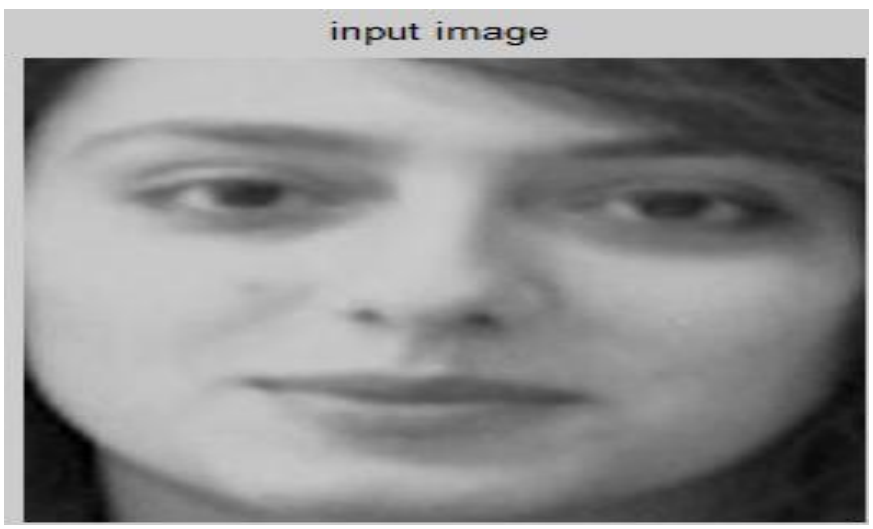

Figure 2: Input Image for Edge Detection

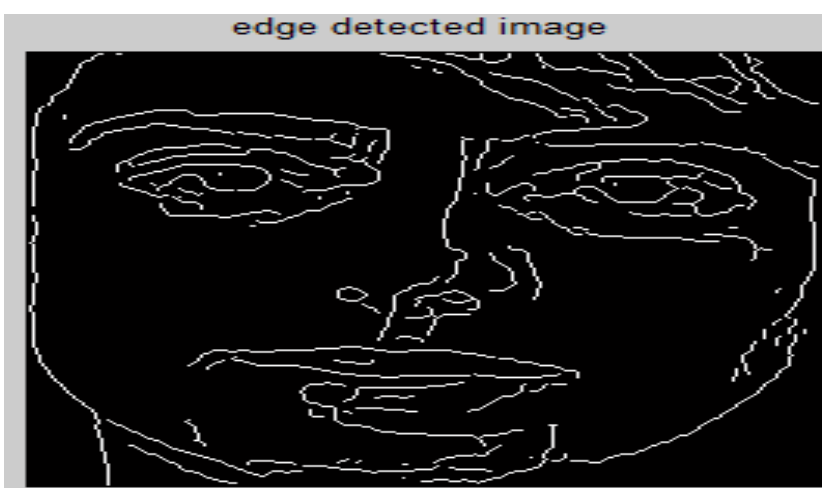

Figure 3: Edge Detection of an Image

\subsection{Feature Extraction}

While working directly on an image the result will be very slow as one small part of a face image can contain a thousands of pixels. Furthermore, all the pixels will not be useful in detection. There can be an underlying structure that further describes the differences between male and female faces better. Thus the feature detection module should be employed here.

Generally there are two types of features presented in the gender classification context they are Geometric-based features and appearance-based features.

- Geometric-based features: Geometric-based features are also called as local features came from psychophysical explorations. They represent highlevel face descriptors likedistances between nose, eyes and mouth, face width, face length, eyebrow thickness and so on

- Appearance-based features: Appearance-based features are also called as global features uses low- 
level information about face image areas based on pixel values. Among appearance-based features the popular ones are

$\checkmark \quad$ various texture features: e.g. Local binary pattern(LBP) Local Directional

$\checkmark \quad$ Pattern(LDP) and Pixel-Pattern-Based Texture Feature (PPBTF)

$\checkmark$ histogram of gradients: scale invariant feature transform

$\checkmark$ coefficients of wavelet transformation of image: e.g. Gabor wavelet and haar wavelet

Both Global and Grid features are combined to extract features. The Global features consist of inter ocular distance, the distance between the lips and the nose tip, the distance between nose tip and the line joining two eyes, the distance between lips and the line joining two eyes. The grid features include the mustache region, lip region, cantus, and nose tip of the facial image.

For extracting the facial features consider the facial image then divide into four equal parts. At each part find the centroid by using the region properties. After finding centroids calculate the distances between the centroids by using the distance formula. Then locate the eyes at upper right and left parts and then locate lips. For locating the chin and nose, we added +45 and -30 to the centroid value of lips so that it can locate chin and nose respectively.

\subsection{Classification}

After extracting all the necessary features, the final task is to decide whether these features represent female or male face. In this work ratios of male and female are calculated and finally found the threshold value. Basing on the four ratios threshold values decision is made whether the given image is a male or a female.

\subsubsection{Facial Features to Be Considered:}

> Inter-ocular distance: The distance between the midpoint of right eye and midpoint of left eye in the face image.

$>$ Lips to Nose: The distance between nose tip and the midpoint of the lips pixel in the facial image.

$>$ Nose to Eyes: The distance between Nose tip to interocular distance in the facial image.

$>$ Lips to Eyes: The distance between lips midpoint to interocular in the facial image.

The following figures gives the ratios of left_eye to right_eye distance, eye to nose, eye to lip and eye to chin distance for feature extraction.[12]

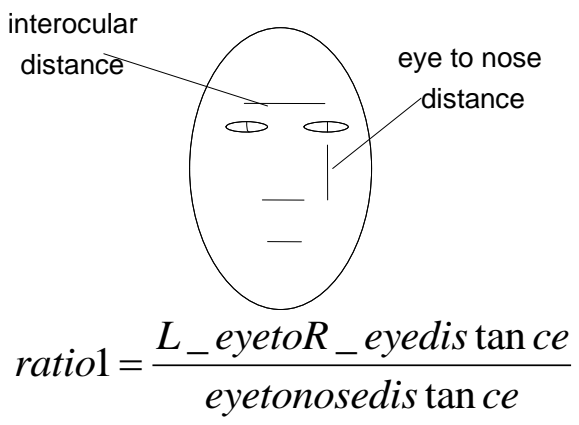

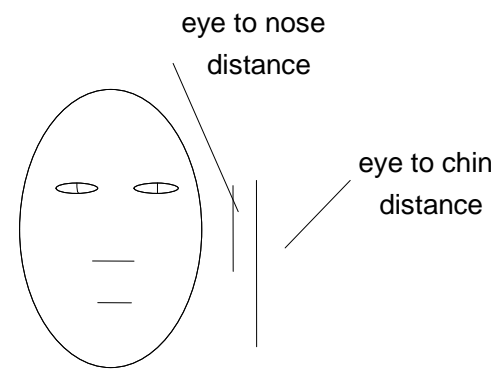

ratio $2=\frac{\text { eyetonosedis } \tan c e}{\text { eyetochindis } \tan c e}$

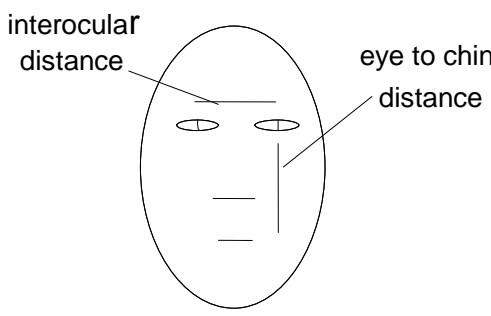

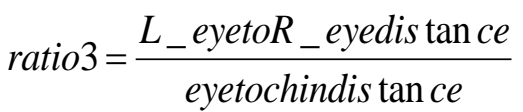

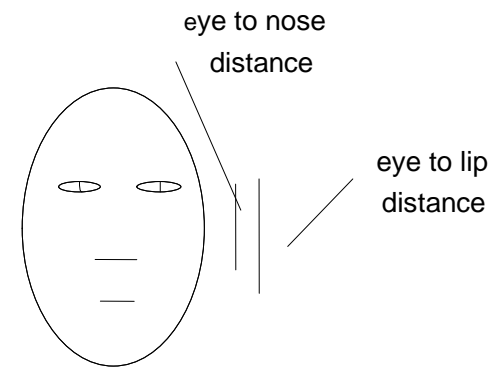

$$
\text { ratio4 }=\frac{\text { eyetonosedis tance }}{\text { eyetonosedis } \tan c e}
$$

Figure 4: Ratios to be considered

After extracting all the necessary features, the final task is to decide whether these features represent female or male face. In this work ratios of male and female are calculated and find the threshold value. Basing on the four ratios set the threshold value by observing number of images and decides whether the given image is a male or a female.

The threshold values for female are ratio1 $>=1.1000 \& \&$ ratio $2>=0.7450 \|$ ratio $3<=1.3714 \& \&$ ratio $4>=$ 0.6404 and for maleratio $1<=1.09 \quad \& \&$ ratio $2<=0.7440$ ratio3 $>=1.3714 \& \&$ ratio $4<=0.6400$. This value is obtained for windows 32 bit. It may vary. For concluding the given image as a male or a female check all the four ratios. If all these values satisfy the above conditions then the given image can be a male or a female. 


\section{RESULTS}

Most of the images are considered from the AT\&T database and trained them. 125 images of both male and female are from the database. The above necessary steps are performed on the trained images and ratios are calculated and are compared with the threshold. First 10 images shows the resultant ratios of male and next 10 images shows the ratios of female images. The testing of face images is performed by using Indian face database. It is examined that the gender recognition rate is $95.6 \%$.

\section{Male images}

1.

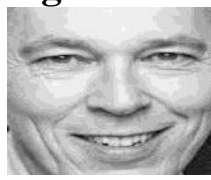

$\begin{array}{llll}0.9078 & 0.5144 & 1.5377 & 0.5666\end{array}$

2.

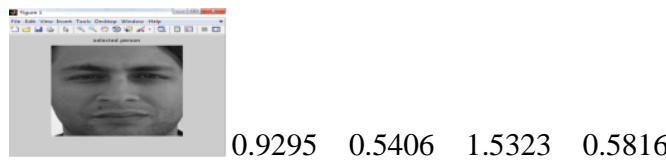

3.

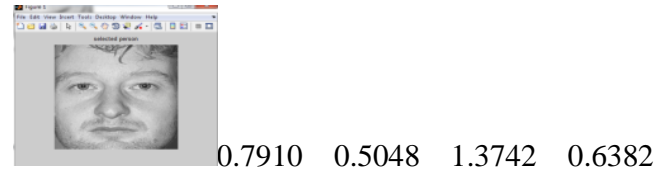

4.

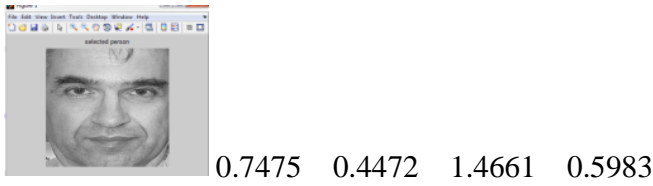

5.

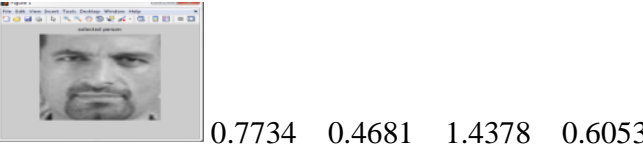

6.

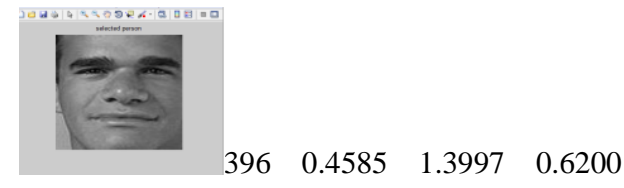

7.

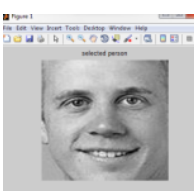

$\begin{array}{llll}0.8415 & 0.5211 & 1.4288 & 0.6193\end{array}$

8.

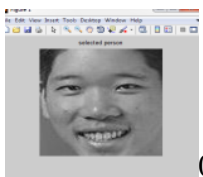

$\begin{array}{llll}0.8198 & 0.4729 & 1.5676 & 0.5769\end{array}$

9.

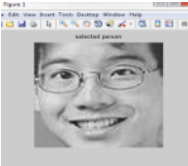

10.

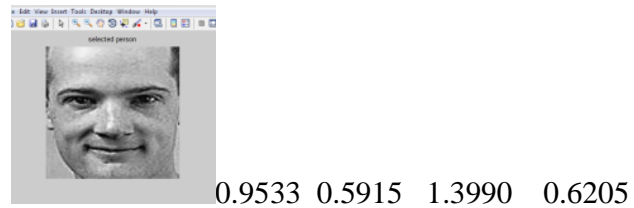

Female images

1.

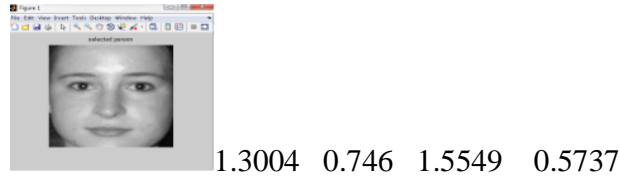

2.

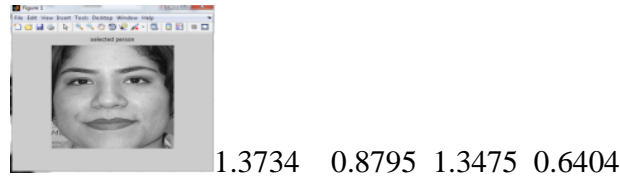

3.

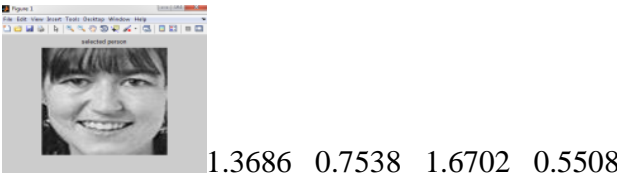

4.

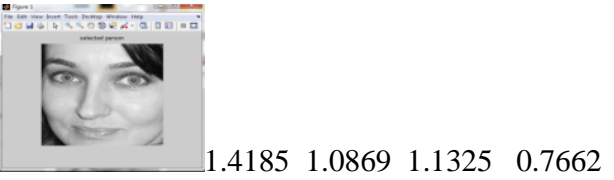

5.

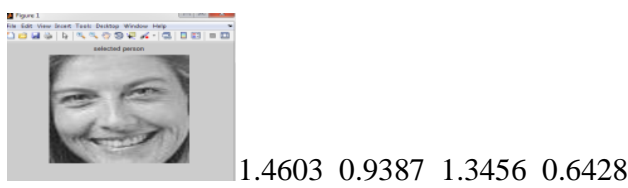

6.

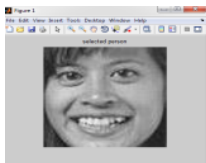

$\begin{array}{llll}1.2017 & 0.816 & 1.2809 & 0.679\end{array}$

7.

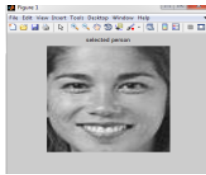

$\begin{array}{llll}1.1876 & 0.7511 & 1.3574 & 0.6324\end{array}$

8.

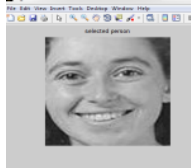

$\begin{array}{llll}1.1762 & 0.7965 & 1.296 & 0.6772\end{array}$

9.

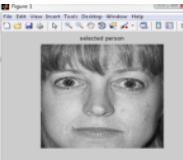

$\begin{array}{llll}1.1521 & 0.7596 & 1.3283 & 0.6593\end{array}$ 
10.

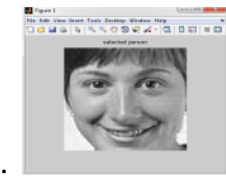

$\begin{array}{llll}1.1699 & 0.7551 & 1.2798 & 0.6454\end{array}$

\section{CONCLUSION}

Detecting gender through a machine is a good achievement in a modern era of computer world. This application is really working in many fields like criminology, medical sciences, in airports for authentication etc. So far gender classification is done for frontal face images only. It can extend to various other poses. Here only four ratios are considered it can extend by considering canthus, forehead, eye brow and moustache regions. It also can be extended for classifying age by analyzing the various facial parts through various mathematical operators.

\section{REFERENCES}

[1] B. Moghaddam and M.H. Yang, "Gender Classification with Support Vector Machines”, Proc. Int'l Conf. Automatic Face and Gesture Recognition, pp. 306-311, Mar.2000.

[2] G. Shakhnarovich, P.A. Viola, and B. Moghaddam, "A Unified Learning Framework for Real Time Face Detection and Classification", Proc. Int'l Conf. Automatic Face and Gesture Recognition, pp. 14-21, 2002.

[3] Ming-Hsuan Yang and Baback Moghaddam, "Support Vector Machines for Visual Gender Classification", Fifteenth International Conference on Pattern Recognition, vol. 1,pp. 5115-5118, 2000.

[4] Baback Moghaddam and Ming-Hsuan, "Learning Gender with Support Faces", IEEE Transactions on Pattern Analysis and Machine Intelligence, vol. 24, no. 5, pp. 707711, May 2002.

[5] Zehang Sun, George Bebis, Xiaoping Yuan, and Sushi J. Louis, "Genetic Feature Subset Selection for Gender Classification": A Comparison Study, IEEE Workshop on Applications of Computer Vision, pp.165-170, 2002.
[6] Roytatsu Iga, Kyoko Izumi, Hisanori Hayashi, GentaroFukano and Testsuya Ohtani, "Gender and Age Estimation from Face Images", International Conference on The Society of Instrument and Control Engineering, pp. 756-761, August, 2003.

[7] Hui-Cheng Lain and Bao-Liang Lu, "Multi-View Gender Classification using Local Binary Patterns and Support Vector Machines", International Conference on Neural Networks, pp. 202-209, 2006.

[8] Jing Wu, W. A. P. Smith and E. R. Hancock, "Gender Classification using Shape from Shading", International Conference on Image Analysis and Recognition, pp. 925934, 2008.

[9] ErnoMakinen and Roope Raisamo, "Evaluation of Gender Classification Methods with Automatically Detected and Aligned Faces", IEEE Transactions on Pattern Analysis and Machine Intelligence, vol. 30, no. 3, pp. 541-547, March 2008.

[10] G. Mallikarjuna Rao, G. R. Babu, G. Vijaya Kumari and N.Krishna Chaitanya, "Methodological Approach for Machine based Expression and Gender Classification, IEEE International Advance Computing Conference”, pp. 1369-1374, 6-7 March 2009.

[11] Hui-Cheng Lain and Bao-Liang Lu, "Age Estimation using a Min-Max modular Support Vector Machine", Twelfth International Conference on Neural Information Processing, pp.83-99,November, 2005.

[12] Rameshk.et.al, "Feature extraction based gender and age estimation" International journal for computer science and Engineering Vol. 02, No. 01s, 2010, 14-23.

[13] V N Prudhvi Raj and Dr T Venkateswarlu, "Denoising of medical ultrasound images using spatial filtering and multiscale Transforms" International Journal of Computer Science \& Information Technology Vol 4, No 6, December 2012 